\title{
A Review on Consensus Clock Synchronization Algorithms
}

\author{
Vincy $\mathbf{V}^{1}$, Dr. Asha T $S^{2}$ \\ PG Student, Communication Engineering, NSS College of Engineering, Palakkad, India ${ }^{1}$ \\ Professor, Electronics and Communication Engineering, NSS College of Engineering, Palakkad, India ${ }^{2}$
}

\begin{abstract}
Recently, Consensus based Clock Synchronization (CCS) algorithms have gained much attention in wireless sensor networks due to its simplicity, distributed nature and robustness. Time synchronization in all networks either wired or wireless is important. It allows for successful communication between nodes on the network. It is, however, particularly vital for wireless networks. Wireless time synchronization is used for many different purposes including location, proximity, energy efficiency, and mobility.
\end{abstract}

Keywords: Consensus Clock Synchronization, Reference clock, Clock offset, Clock skew

\section{INTRODUCTION}

Wireless networks are an increasingly important medium for distributed computation. Time synchronization is essential in sensor networks since due to change in external environment the inbuilt clock may vary. Each sensor node has an inbuilt clock, which is set to a common time before the deployment. After the deployment of the sensor nodes, their clock time might change during the operation due to different climatic conditions like temperature and humidity, as the sensors may not experience the same conditions, when they are deployed over a larger geographical area [16]

The various protocols for clock synchronization are [1-16] Some protocols uses external reference clock for synchronization [1-5]The main disadvantage of this method is, Each time the clock value is updated at a node, there is an inherent error, which is passed on to other nodes as this propagates, resulting in greater accumulative error by the time the network synchronization is reached. In some protocols [10-14] the modifications of the clock is done recursively to achieve clock synchronization. These protocols converge to the network synchronization time with greater accuracy and lesser accumulative effect but with higher computation overhead. Another type of synchronization protocols are consensus based protocols[6-10]. This type of protocols does not use external or internal reference clock. The network synchronization time is set, based on the consensus between the nodes. The network synchronization time could be set to either the fastest clock of the network or to the average time of the nodes in a network. The consensus based protocols are efficient, but have greater communication overhead.

\section{CONSENSUS BASED CLOCK SYNCHRONIZATION}

Consensus time synchronization technique does not need to know the topology structure of network to synchronize. It depends on the exchange of time messages between nodes to convergent the local clocks on nodes to the same reference clock. The consensus time synchronization technique is robust and has good scalability. In Consensus synchronization the clock value is not taken from the reference node but the clock value is taken based upon consensus with all the nodes in the network. All the nodes may agree to adapt to the fastest clock in the network as in MTS [6] or to the average time of the clocks of the network as in ATSP [7].

A. Maximum value based consensus approach.

Compared with the traditional tree-based or reference (root)-based time synchronization algorithm, MTS has the advantage of being fully distributed, asynchronous, robust to packet drop, sensor node failure and new node joining, and it is adaptive to time-varying communication topology. Different from traditional time synchronization protocols, such as FTSP, MTS does not require setting a root node or building up a tree topology in advance, which largely reduces the implementing costs and enhances system robustness. Moreover, MTS can conduct both skew and offset compensation at the same time and complete them simultaneously in finite time[6].

The basic idea of our approach is to drive the logical clocks to the maximum value among all nodes so that the network can achieve time synchronization. . In the algorithm, each node i periodically broadcasts a packet containing its local hardware clock reading $T_{i}(t)$ and its current relative logical clock skew $a_{i}(t)$ and the offset $b_{i}(t)$, without requiring any 


\section{International Advanced Research Journal in Science, Engineering and Technology}

Vol. 6, Issue 3, March 2019

feedback information from its neighbors. For each neighbor node, a pair of hardware clock readings needs to be stored by node i. Since the protocol drives all nodes throughout the network to approach the maximum hardware clock, that is, maximum-consensus, we name the protocol as Maximum Time Synchronization. According to the protocol, the skew and offset compensations are conducted simultaneously and completed at the same time. MTS algorithm converges within a finite time and the expected convergence time of MTS is also finite[6].

\section{B. Average Time Synchronization(ATS).}

ATS algorithm is based on a cascade of two consensus algorithms, whose main idea is averaging local information. The Average Time Synchronization protocol includes three main parts: the relative skew estimation, the skew compensation, and the offset compensation.

\section{Relative Skew Estimation.}

This part of the protocol is concerned with deriving an algorithm to estimate for each clock $i$ the relative skew with respect to its neighbors $\mathrm{j}$. Every node $\mathrm{i}$ tries to estimate the relative skews with respect to its neighbor nodes $\mathrm{j}$ This is accomplished by writing the current local time of node $\mathrm{j}$ into a broadcast packet, then the node $\mathrm{i}$ that receives this packet immediately records its own local time and estimate the relative skew[7].

\section{Skew Compensation.}

This part of the algorithm is the core of the Average Time Synchronization protocol, as it forces all the nodes to converge to a common virtual clock rate, $\alpha$, as. The main idea is to use a distributed consensus algorithm based only on local information exchange. In consensus algorithms any node keeps its own estimate of a global variable, and it updates its value by averaging it with the estimates of its neighbors [7].

\section{Offset Compensation.}

According to the previous analysis, after the skew compensation algorithm is applied, the virtual clock estimators have all the same skew, i.e. they run at the same speed. At this point it is only necessary to compensate for possible offset errors. Each node compute the instantaneous estimated clock difference and try to update its offset in order to reduce the difference[7].

\section{Clock Synchronization using Least common multiple(CSLCM)}

The proposed CSLCM enables the nodes to reach a network synchronization time by calculating the least common multiple of their Clock Time Period (CTP). The network is organized into clusters and every node reaches the network synchronization time using its own CTP. This method consists of 4 steps; Cluster formation, Clock synchronization of nodes, Intra cluster synchronization and Inter cluster synchronization [16].

\section{Block Diagram of CSLCM.}

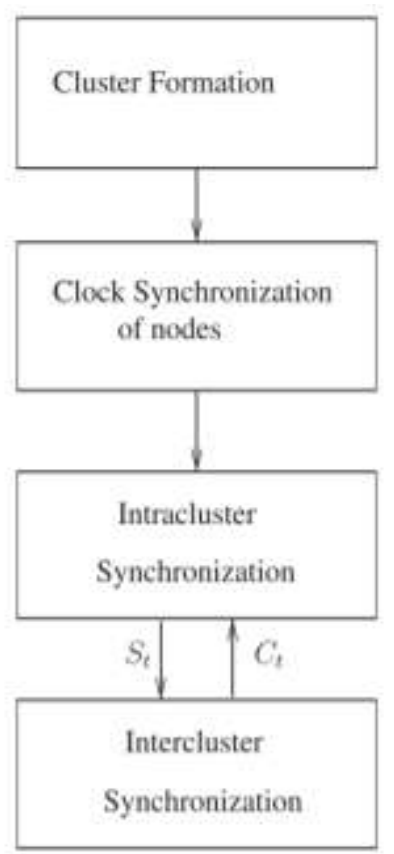

Fig 1. Block diagram of CSLCM[16] 


\title{
International Advanced Research Journal in Science, Engineering and Technology
}

\author{
Vol. 6, Issue 3, March 2019
}

The block diagram for CSLCM is shown in Fig. 1. There are 2 steps in the proposed CSLCM method. The first step in CSLCM is to organize the randomly deployed nodes into clusters. The second step is to synchronize the nodes. The synchronization process carried among the cluster members is termed as intracluster synchronization and the synchronization among the cluster heads is termed as intercluster synchronization. The election of cluster head based on the maximum weight. In intracluster synchronization, each cluster applies CSLCM method to synchronize to a synchronization time. The clock time period CTP of every node in the cluster is passed on to the cluster head. The cluster head collects the CTP from its cluster members and broadcasts it within the cluster. All the cluster members know the CTP of each other within the cluster. Every node calculates the Least Common Multiple (LCM) of CTPs. So all the cluster members of a cluster arrive at a common value(LCM), which is referred as synchronization time. In intercluster synchronization, we make an assumption that the cluster heads can reach the base station. The cluster heads, transmit their intracluster synchronized values to the base station. The base station collects these values and broadcasts to the cluster heads. The cluster heads calculate the new synchronization time. The new value is again broadcasted by the cluster heads to their cluster members. clusters easily change their time to new synchronization time, which is the global time for network synchronization[16].

\section{CONCLUSION}

The consensus based time synchronization techniques have been reviewed in this paper. Three consensus algorithms has been reviewed; Maximum value based consensus approach, Average time synchronization and synchronization based on LCM .Maximum value based approach has fast convergence speed but overhead is considerably more compared to other two algorithms. ATS is robust to packet drop and sensor node failure, and it is adaptive to clock drifts and changes on the communication topology. The synchronization error is reduced in ATS. CSLCM is simple to implement. The convergence time is less in case of CSLCM. The main advantage of CSLCM is considerable reduction in synchronization error and also the overheads is reduced.

\section{REFERENCES}

[1]. Ma Tao, Xu Zhanqi, Hempel Michael, Peng Dongming, Sharif Hamid. Performance analysis of a novel low complexity high precision timing synchronization method for wireless sensor networks. IEEE Trans Wireless Communication2014.

[2]. Huang Ge, Zomaya Albert Y, Delicato Flavia C, Pires Paulo F. Long term and large scale time synchronization in wireless sensor networks. Computer Communication 2014.

[3]. Huang Ge, Zomaya Albert Y, Delicato Flavia C, Pires Paulo F. An accurate on-demand time synchronization protocol for wireless sensor networks. Parallel Distributed Computation 2012.

[4]. Ganeriwal S, Kumar R, Srivastava MB. Timing-sync protocol for sensor networks. In: Proceedings of the 1st international conference on embedded networked sensor systems-ACM; 2003.

[5]. Huang Hai-ping, Wang Ru-chuan, Li 3 Liu, Sun Li-juan, Li Wen-feng. Time synchronization algorithm of wireless sensor networks based on data aggregation tree. Sci Direct 2010.

[6]. He Jianping, Chang Peng, Shi Ling, Chan Jiming, Sun Youxian. Time synchronization in wireless sensor networks: a max-value based consensus approach. IEEE Trans Autom Control 2014.

[7]. Wu Jianshe, Jiao Licheng, Ding Ranran. Average time synchronization in wireless sensor networks by pairwise messages. Compution Communication 2012.

[8]. Garone Emanuele, Gasparri Andrea, Lamonaca Francesco. Clock synchronization protocol for wireless sensor networks with bounded communication delays. Automatica 2015.

[9]. Panigrahi Niranjan, Khilar Pabitra Mohan. An evolutionary based topological optimization strategy for consensus based clock synchronization protocols in wireless sensor network. Swarm Evol Comput 2015.

[10]. Panigrahi Niranjan, Khilar Pabitra Mohan. Multi-hop consensus time synchronization algorithm for sparse wireless sensor network: a distributed constraint-based dynamic programming approach. Ad-Hoc Network 2017.

[11]. Yildirim Kasim Sinan, Carli Ruggero, Schenato Luca. Adaptive control based clock synchronization in wireless sensor networks. In: European control conference (ECC); 2015.

[12]. chen Zhenping, Li Dequan, Huang Yourui, Tang Chaoli. Event triggered communication for time synchronization in Wireless sensor networks. Neurocomputing 2016

[13]. Chovanec Michal, Puchyova Jana, Hudik Martin, Kochlan Michal. Universal synchronization algorithm for Wireless sensor networks -FUSA algorithm. In: Proceedings of the 2014 federated conference on computer science and information system, vol. 2; 2014.

[14]. Watwe S, Hansdah RC. Improving the energy efficiency of a clock synchronization protocol for wireless sensor networks using TDMA based MAC protocol. In: IEEE 29th international conference on advanced information networking and applications; 2015.

[15]. Khurana Manju, Thalore Ranjana, Raina Vikas, Jha Manish Kumar. Improved time synchronization in ML-MAC for WSN using relay nodes. Int J Electron Commun (AEU) 2015.

[16]. Nikhath Tabassum, Geetha D. Devanagavi, Rajashekhar C. Biradar. Clock synchronization in wireless sensor networks using least common multiple. Int. J. Electron. Commun. (AEÜ) 2017. 